\title{
Qualidade de vida entre estudantes de Odontologia e o rendimento acadêmico
}

\section{Quality of life among dental students and their academic performance}

\author{
Ana Katarina Martins \\ Universidade Estadual de Ponta Grossa - UEPG - Ponta Grossa - Brasil \\ k.anaa@hotmail.com \\ Sabrina Brigola \\ Universidade Estadual de Ponta Grossa - UEPG - Ponta Grossa - Brasil \\ sabrinabrigola@hotmail.com \\ Daniela Huller \\ Universidade Estadual de Ponta Grossa - UEPG - Ponta Grossa - Brasil \\ danihuller@hotmail.com \\ Márcia Thaís Pochapski \\ Universidade Estadual de Ponta Grossa - UEPG - Ponta Grossa - Brasil \\ mpochapski@gmail.com \\ Fábio André dos Santos \\ Universidade Estadual de Ponta Grossa - UEPG - Ponta Grossa - Brasil \\ fasantos@uepg.br
}

\section{Resumo}

A avaliação da qualidade de vida em estudantes de graduação em Odontologia é uma importante ferramenta para o planejamento curricular e institucional. $O$ objetivo do presente trabalho foi avaliar a qualidade de vida de estudantes de graduação em Odontologia e o impacto no desempenho acadêmico. Participaram deste estudo, 86 estudantes matriculados nas $2^{\mathrm{a}}$ e $3^{\mathrm{a}}$ séries de um Curso de Odontologia. A avaliação do desempenho acadêmico foi realizada por meio das notas obtidas na disciplina de Periodontia Pré-clínica, ministrada simultaneamente nas duas séries. Para a avaliação da qualidade de vida foi utilizada a versão em Português do questionário WHOQOL-Bref. A análise de dados foi realizada com teste $t$ de student, qui-quadrado e regressão linear múltipla. Os resultados mostraram diferenças na qualidade de vida entre os gêneros feminino e masculino (domínios "físico" e "psicológico"). Não foram encontradas diferenças significativas entre os acadêmicos da diferentes séries e gêneros. $O$ domínio "relações sociais" foi relacionado com o desempenho acadêmico. A partir dos resultados obtidos foi possível concluir que os homens apresentam melhores indicadores de qualidade de vida que as mulheres. Não foram encontradas diferenças no rendimento acadêmico dos alunos, sendo que o domínio "relações sociais" teve relação com a performance do estudante.

Palavras-chave: qualidade de vida, educação em odontologia, estudantes de odontologia, desempenho acadêmico. 


\section{Abstract}

The quality of life evaluation in undergraduate dental students is an important tool for curricular and institutional planning. The objective of this present study was to evaluate the quality of life of undergraduate dental students and its impact on their academic performance. This study included 86 students enrolled in the 2nd and 3rd grades of a dental school. The evaluation of the academic performance was performed by grade means obtained in the Preclinical Periodontics subject which taught simultaneously in both series. The Portuguese version of the WHOQOL-Bref questionnaire was used to evaluate the quality of life. Data analysis was performed with student $t$-test, chi-square and multiple linear regression. The results showed differences in quality of life between females and males ("physical" and "psychological" domains). No significant differences were found between students of different series and genders. The "social relations" domain was related to academic performance. From the results, it was possible to conclude that men present better indicators of quality of life than women. No differences were found in the students' academic performance, and the "social relations" domain was related to the student's performance.

Keywords: quality of life, dental education, dental students, academic performance.

\section{Introdução}

A qualidade de vida (QV) é um conceito complexo, uma vez que a interpretação depende do ponto de vista de cada indivíduo, é também multidimensional, dada a influência de fatores relacionados à educação, economia e aspectos socioculturais (FLECK et al., 2000). A Organização Mundial de Saúde (OMS) define QV como a "percepção do indivíduo de sua posição na vida, no contexto da cultura e sistemas de valores nos quais ele vive e em relação aos seus objetivos, padrões e preocupações" (COSTA et al., 2018; LANGAME et al., 2016). A QV dos profissionais de saúde é um tema de interesse entre educadores e pesquisadores (SAUPE et al., 2004; ZHANG et al., 2012). Os efeitos da QV de acadêmicos de graduação das ciências médicas, tem sido estudado em diferentes países (ANDRE; PIERRE; MCANDREW, 2017; CRUZ et al., 2018; MORITZ et al., 2016; MOURA et al., 2016; SERINOLLI; NOVARETTI, 2017).

Vários fatores podem interferir na QV de estudantes universitários, incluindo o estilo de vida, condições econômicas, relações sociais, padrão de sono, aspectos psicológicos, bem como, o competitivo ambiente universitário (CHAZAN; CAMPOS; PORTUGAL, 2015; GAN; YUEN LING, 2019). Na área da saúde, estudos mostram que estudantes de Medicina apresentam problemas psicológicos e emocionais como estresse, ansiedade e depressão, sendo estes, mais relacionados com a mulheres, e estas características podem comprometer o desempenho acadêmico (GAN; YUEN LING, 2019; MALIBARY et al., 2019; SHAREEF et al., 2015; SOLIS; LOTUFO-NETO, 2019). Pesquisas mostram (ABOSHAIQAH; CRUZ, 2019; CRUZ et al., 2018; MORITZ et al., 2016; MOURA et al., 2016; SAUPE et al., 2004) que estudantes de Enfermagem, também apresentam variações na $Q V$, sendo que fatores como o grau de desenvolvimento do país em que os dados foram coletados, bem como as condições sócio-econômicas, influenciam diretamente os resultados.

Há poucos estudos envolvendo alunos de Odontologia (ANDRE et al., 2017; RODRIGUES et al., 2019). O curso de Odontologia apresenta algumas características específicas, sendo que nos primeiros anos, há um amplo conteúdo teórico com atividades pré-clínicas, que incluem conceitos básicos da Odontologia e o desenvolvimento da habilidade manual, necessária para a atividade prática do dentista. Fatores associados com esta fase, como a carga horária teórica, competição entre os alunos, podem afetar a QV e a performance acadêmica. Nos últimos ano do curso, os estudantes executam as 
atividades com o atendimento clínico de pacientes, nesta fase, os fatores relacionados com o aumento do estresse, estão associados com as responsabilidades com os pacientes, necessidade de boa habilidade manual e expectativas após a formatura. Esses fatores, podem ser fontes de desordens psicológicas que podem impactar o desempenho dos alunos (ANDRE et al., 2017; RODRIGUES et al., 2019).

Assim, estudos que avaliam a QV em estudantes de graduação em Odontologia, são importantes no planejamento curricular e institucional, para a promoção e atenção à saúde dos acadêmicos. Tais ações, podem contribuir para a melhor performance acadêmica e por consequência ter boas repercussões na vida profissional do futuro cirurgião dentista. Desta forma, o objetivo do presente trabalho foi avaliar a QV de estudantes de graduação em Odontologia e o impacto no desempenho acadêmico.

\section{Material e método}

Participaram deste estudo observacional, todos os acadêmicos regularmente matriculados nas $2^{\mathrm{a}}$ e $3^{\mathrm{a}}$ séries do Curso de Odontologia no segundo semestre de 2017 da Universidade Estadual de Ponta Grossa. A população do estudo foi composta por 103 universitários. O desenvolvimento do trabalho foi realizado na disciplina de Periodontia Pré-clínica, que devido a transição curricular, foi ministrada simultaneamente nas duas séries.

Como critérios de inclusão, o aluno deveria estar devidamente matriculado em uma das disciplinas e concordado com termo de consentimento livre esclarecido (Parecer CEP: 2.133.732; CAAE: 69794617.6.0000.5689). Estudantes que já haviam cursado a disciplina anteriormente e foram reprovados, foram excluídos do estudo.

Os alunos das duas séries receberam durante o semestre os mesmos conteúdos ministrados sempre pelo mesmo professor. A avaliação do desempenho acadêmico foi realizada por meio das notas obtidas em duas avaliações dissertativas ( $1^{\circ}$ e $2^{\circ}$ bimestres), bem como, a média final.

Para a avaliação da QV foi utilizada a versão em Português do questionário WHOQOL-Bref que contém 26 questões, das quais duas são relacionadas a QV geral (percepção da QV e satisfação com a saúde) e 24 questões relacionadas aos domínios físico (dor e desconforto; energia e fadiga; sono e repouso; mobilidade; atividades da vida cotidiana; dependência de medicação ou tratamentos; capacidade de trabalho), psicológico (sentimentos positivos; pensar, aprender, memória e concentração; autoestima; imagem corporal e aparência; sentimentos negativos; espiritualidade, religião e crenças pessoais), social (relações pessoais; suporte social; atividade sexual) e meio ambiente (segurança física e proteção; ambiente no lar; recursos financeiros; cuidados com a saúde e sociais; oportunidades de adquirir novas informações e habilidades; recreação e lazer; transporte; ambiente físico). Todas as questões foram respondidas em uma escala Likert de cinco pontos (FLECK et al., 2000). Para fins de comparação com estudos sobre a temática, consideramos as duas questões sobre a QV geral e os domínios do instrumento, sendo que para estes, o escore final foi transformado em uma escala de 0 a 100 pontos, na qual valores mais próximos de zero indicam pior QV enquanto os mais próximos de 100, melhor QV (PEDROSO et al., 2010). O WHOQOLbref é um instrumento transcultural, validado para o Português, apresenta boa consistência e reprodutibilidade (CHAZAN et al., 2015; FLECK et al., 2000).

As características gerais da amostras foram avaliadas com estatística descritiva e analítica (teste $\mathrm{t}$ de student e qui-quadrado). Os dados obtidos com o WHOQOL-Bref foram comparados com o teste $t$ de student para amostras não pareadas. A relação entre a série, idade, gênero e QV com o desempenho acadêmico foi realizado com o modelo de regressão linear múltipla padrão. A normalidade dos dados foi verificada com o teste de Kolmogorov \& Smirnov e confirmada com os coeficientes de assimetria e kurtose. O nível 
de significância adotado foi de $5 \%(\alpha=0,05)$. Todos os cálculos foram realizados com 0 pacote estatístico SPSS® (Statistical Package for the Social Science) versão 21.0 (SPSS Inc Chigaco Illinois USA).

\section{Resultados}

Do total de 103 alunos matriculados nas $2^{a}$ e $3^{a}$ séries do Curso de Odontologia, 86 responderam todas as questões do questionário ( $84 \%$ do total).

Tabela 1. Características gerais da amostra de alunos do Curso de Odontologia da Universidade Estadual de Ponta Grossa.

\begin{tabular}{cccc}
\hline \multirow{2}{*}{ Variáveis } & \multicolumn{2}{c}{ Série } & \multirow{2}{*}{ Valor de P } \\
\cline { 2 - 3 } & $2^{\circ}$ Ano & $3^{\circ}$ Ano & $0,023^{\mathrm{s}}$ \\
\hline Idade em anos (média $\pm \mathrm{DP})^{\ddagger}$ & $19,8 \pm 1,7$ & $20,7 \pm 1,7$ & \\
Gênero $(\%)^{\dagger}$ & & & $0,172^{\mathrm{ns}}$ \\
Feminino & $33(85)$ & $34(72)$ & \\
Masculino & $6(15)$ & $13(28)$ & \\
Total & $39(100)$ & $47(100)$ & \\
\hline
\end{tabular}

ft student; ${ }^{\dagger} \chi^{2}$; ssignificativo; ns não significativo

Nota: 17 alunos foram excluídos

Fonte: Autores (2017)

A idade média foi de $20,2 \pm 1,7$ anos, sendo que $72 \%$ eram do gênero feminino (Tabela 1).

Tabela 2. Qualidade de vida e avaliação da vida acadêmica em acadêmicos de odontologia de acordo com o gênero e série.

\begin{tabular}{|c|c|c|c|c|c|c|}
\hline \multirow[b]{2}{*}{$\begin{array}{l}\text { Qualidade de vida } \\
\text { Whoqol-bref }\end{array}$} & \multicolumn{2}{|c|}{ Gênero } & \multirow{2}{*}{$\begin{array}{c}\text { Valor } \\
\text { de } \\
P\end{array}$} & \multicolumn{2}{|c|}{ Série } & \multirow{2}{*}{$\begin{array}{c}\text { Valor } \\
\text { de } \\
P\end{array}$} \\
\hline & $\begin{array}{c}\text { Feminino } \\
\mathrm{n}=67\end{array}$ & $\begin{array}{c}\text { Masculino } \\
n=19\end{array}$ & & $\begin{array}{c}2^{\circ} \text { Ano } \\
\mathrm{n}=39\end{array}$ & $\begin{array}{l}3^{\circ} \text { Ano } \\
n=47\end{array}$ & \\
\hline $\begin{array}{l}\text { Percepção da Qualidade } \\
\text { de Vida }\end{array}$ & $3,7 \pm 0,7$ & $4,0 \pm 0,8$ & 0,180 & $3,9 \pm 0,8$ & $3,7 \pm 0,7$ & 0,376 \\
\hline Satisfação com a Saúde & $3,4 \pm 1,0$ & $3,6 \pm 1,0$ & 0,369 & $3,4 \pm 1,0$ & $3,4 \pm 1,0$ & 0,962 \\
\hline Domínio Físico & $55,9 \pm 15,3$ & $66,2 \pm 14,8$ & $0,011^{*}$ & $56,1 \pm 16,5$ & $59,9 \pm 14,9$ & 0,263 \\
\hline Domínio Psicológico & $55,9 \pm 15,8$ & $66,0 \pm 11,9$ & $0,012^{*}$ & $58,7 \pm 16,0$ & $57,7 \pm 15,3$ & 0,782 \\
\hline Domínio Relações Sociais & $63,6 \pm 20,1$ & $69,3 \pm 13,9$ & 0,261 & $65,8 \pm 19,8$ & $64,0 \pm 19,5$ & 0,673 \\
\hline Domínio Meio Ambiente & $61,8 \pm 13,9$ & $61,8 \pm 17,6$ & 0,982 & $64,0 \pm 13,9$ & $59,9 \pm 15,2$ & 0,197 \\
\hline
\end{tabular}

t student; ${ }^{*}$ diferença significativa

Nota: 17 alunos foram excluídos

Fonte: Autores (2017)

A tabela 2 mostra que houve diferenças na QV entre os gêneros feminino e masculino, considerando os domínios "físico" e "psicológico". Quando os dados foram agrupados de acordo com a série, não foram encontradas diferenças significativas entre os acadêmicos. 
Figura 1. Distribuição das notas do obtidas de acordo com o gênero $(A)$ e série (B). Linhas representam a média e o desvio padrão; Pontos representam cada aluno. Comparação entre os grupos em cada bimestre, diferenças não significativas $(p>0,05)$. Teste $t$ de student não pareado. Nota: 17 alunos foram excluídos
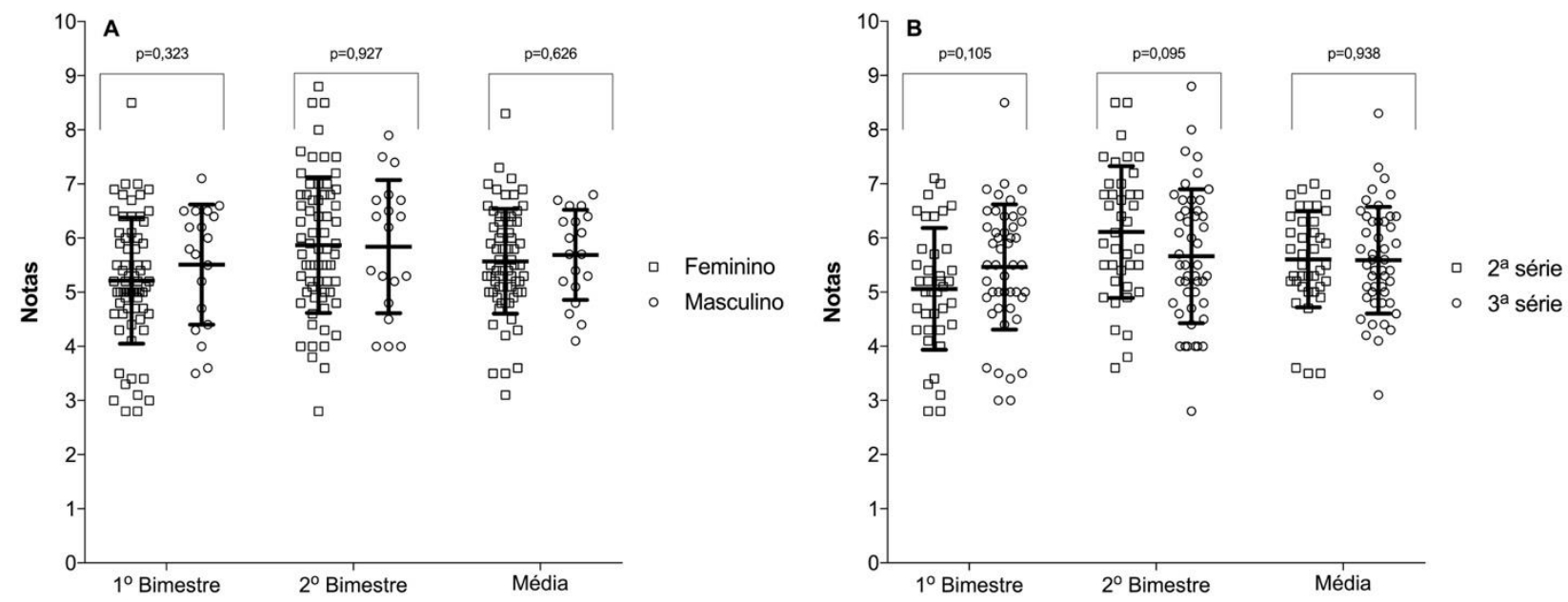

Fonte: Autores (2017)

Não foram observadas diferenças significativas entre as notas dos $1^{\circ}$ e $2^{\circ}$ bimestres, bem como na média final entre os alunos, considerando gênero e série (Figura 1).

A tabela 3 mostra o resultado da análise regressão múltipla, dentro dos diferentes fatores que poderiam ter influência no desempenho acadêmico, encontramos correlação com o domínio "relações sociais".

Tabela 3. Análise de regressão linear múltipla padrão para verificar as correlações entre as variáveis independentes do estudo e o desempenho acadêmico (notas).

\begin{tabular}{lcccccc}
\hline \multirow{2}{*}{ Variáveis Independentes } & \multicolumn{7}{c}{ Variáveis Dependentes } \\
\cline { 2 - 7 } & \multicolumn{2}{c}{ Nota $1^{\circ}$ Bimestre } & Nota $2^{\circ}$ Bimestre & \multicolumn{2}{c}{ Média } \\
& $\beta$ & $\mathrm{P}$ & $\beta$ & $\mathrm{P}$ & $\beta$ & $\mathrm{P}$ \\
\hline Gênero & 0,112 & 0,335 & 0,048 & 0,677 & 0,095 & 0,403 \\
Série & 0,222 & 0,053 & $-0,159$ & 0,161 & 0,036 & 0,748 \\
Idade & $-0,116$ & 0,319 & $-0,201$ & 0,085 & $-0,202$ & 0,078 \\
Qualidade de vida Whoqol-bref & & & & & & \\
$\quad$ Percepção da Qualidade de & 0,020 & 0,898 & $-0,105$ & 0,492 & $-0,058$ & 0,702 \\
$\quad$ Vida & & & & & & \\
$\quad$ Satisfação com a Saúde & $-0,027$ & 0,846 & 0,009 & 0.949 & $-0,012$ & 0,931 \\
$\quad$ Domínio Físico & $-0,087$ & 0,588 & 0,150 & 0,346 & 0,043 & 0,785 \\
$\quad$ Domínio Psicológico & 0,119 & 0,431 & $-0,013$ & 0,933 & 0,068 & 0,645 \\
$\quad$ Domínio Relações Sociais & $-0,258$ & $0,032^{*}$ & $-0,243$ & $0,042^{*}$ & $-0,324$ & $0,006^{*}$ \\
$\quad$ Domínio Meio Ambiente & 0,216 & 0,148 & 0,142 & 0,336 & 0,231 & 0,114 \\
\hline *significativo (p<0,05) & & & & & &
\end{tabular}

Nota: 17 alunos foram excluídos

Fonte: Autores (2017)

\section{Discussão}

Os resultados não apresentaram diferenças entre o gênero e a série, considerando a percepção da QV e a satisfação com a saúde. O valor para a percepção da QV $(3,8)$ foi maior que do que a satisfação com a saúde $(3,4)$, sendo que $74 \%$ consideraram a QV como boa ou muito boa e $59 \%$ declararam estarem satisfeitos ou muito satisfeitos com a própria saúde. A literatura mostra resultados semelhantes em estudantes da área da saúde (Enfermagem e Medicina) com valores para a percepção da QV variando de 3,7 a 
4,0 e para a percepção da saúde entre 3,6 a 3,9. De acordo com os estudos, a QV foi considerada boa ou muito boa por 57 a $73 \%$ dos indivíduos e a satisfação com a saúde de 51 a 68\% (CRUZ et al., 2018; GAN; YUEN LING, 2019; MALIBARY et al., 2019; MOURA et al., 2016; SAUPE et al., 2004). Porém, de acordo com os nossos resultados, deve-se considerar que $8 \%$ consideraram a QV ruim ou muito ruim e $17 \%$ se declararam muito insatisfeitos ou insatisfeitos coma própria saúde. Assim, é necessária uma maior atenção aos alunos que declaram baixa QV e percepção da saúde, devendo os cursos de graduação, disponibilizar programas que ofereçam suporte a estes indivíduos (SAUPE et al., 2004).

Os resultados mostraram que os domínios "relações sociais" e "meio ambiente" tiveram os maiores valores, por outro lado, os domínios "psicológico" e "físico" foram os mais baixos. Esses resultados foram semelhante à outros estudos envolvendo estudantes da área da saúde como Enfermagem, Medicina e Odontologia, que mostraram que o domínio "relações sociais" (ABOSHAIQAH; CRUZ, 2019; ANDRE et al., 2017; COSTA et al., 2018; MOURA et al., 2016) e "meio ambiente" (MALIBARY et al., 2019) também tiveram valores elevados. No entanto, outros estudos mostraram que os domínios "psicológico" e "físico" tiveram os maiores valores (CRUZ et al., 2018; GAN; YUEN LING, 2019). A principais razões para essas diferenças estão relacionadas as características sócio-cultural dos vários países, cursos, currículos e até mesmo das instituições onde os dados foram coletados (ABOSHAIQAH; CRUZ, 2019; ANDRE et al., 2017; CRUZ et al., 2018; SOLIS; LOTUFO-NETO, 2019; ZHANG et al., 2012).

Quando comparam-se os gêneros masculino e feminino, observamos diferenças entre os grupos para os domínios "físico" e "psicológico", com melhores indicadores para os homens. Outros trabalhos também mostraram que estudantes do gênero feminino apresentaram menores valores para os domínios de QV do WHOQOL-bref (GAN; YUEN LING, 2019; MORITZ et al., 2016; RODRIGUES et al., 2019; SERINOLLI; NOVARETTI, 2017; SHAREEF et al., 2015; ZHANG et al., 2012). Porém, também foi possível encontrar estudos em que não foram observadas diferenças entre os gêneros (MALIBARY et al., 2019; SOLIS; LOTUFO-NETO, 2019). As principais razões para as diferenças entre homens e mulheres em relação aos domínios de QV, podem estar relacionadas aos aspectos sócio-culturais das amostras envolvidas nos estudos, e também pelas mulheres serem mais sensíveis e susceptíveis à alterações de humor devido às variações hormonais (GAN; YUEN LING, 2019; MORITZ et al., 2016; VICTOR et al., 2019). Outro aspecto que deve ser considerado, é que nossa amostra foi composta predominantemente por estudantes do gênero feminino $(72 \%)$, provavelmente se a amostra fosse equilibrada entre os gêneros, os resultados poderiam ter sido diferentes. Os cursos na área da área da saúde apresentam de maneira geral, predomínio do gênero feminino, variando de 60 a 90\% (CHAZAN et al., 2015; COSTA et al., 2018; MALIBARY et al., 2019; MORITZ et al., 2016; MOURA et al., 2016; RODRIGUES et al., 2019; SAUPE et al., 2004). Porém, outros estudos mostram cursos como os de Medicina e Odontologia com uma proporção mais equilibrada entre homens e mulheres, o que pode contribuir para resultados mais similares entre os valores obtidos (ANDRE et al., 2017; MALIBARY et al., 2019; SOLIS; LOTUFO-NETO, 2019),

Em relação ao desempenho acadêmico, não encontramos diferenças significativas entre os gêneros e séries nas notas obtidas durante o semestre em que a pesquisa foi desenvolvida. Participaram do estudo 86 estudantes que representaram $30 \%$ do total de alunos matriculados no curso de Odontologia. Os motivos para a escolha da $2^{\mathrm{a}}$ e $3^{\mathrm{a}}$ séries, foi devido à transição do currículo, em que a disciplina de Periodontia Pré-clínica foi ministrada simultaneamente nas duas séries. Outra questão importante a ser considerada, é que nas $1^{\underline{a}}$ e $2^{\underline{a}}$ séries, o Curso de Odontologia apresenta aulas teóricas e práticas em laboratório. A $3^{\underline{a}}$ série marca a transição para a etapa com atendimento clínico de pacientes, em que são incluídas disciplinas como dentística restauradora, 
endodontia, cirurgia bucal e clínica integrada. A transição para a prática clínica pode envolver maior ansiedade e pressão nos alunos, o que poderia impactar na QV e no desempenho (RODRIGUES et al., 2019; ZHANG et al., 2012). Poderíamos esperar um melhor desempenho dos alunos da $3^{\text {a }}$ série, visto que estes já tiveram uma maior número de disciplinas em comparação à $2^{a}$ série, porém isso não foi confirmado. Em alunos de Medicina, a transição para a fase clínica pode impactar na QV em comparação com outras etapas do curso de Medicina, uma vez que os indicadores de QV diminuem nos períodos finais do curso (CHAZAN et al., 2015; ZHANG et al., 2012). Porém, essa característica não foi vista em acadêmicos de Odontologia (ANDRE et al., 2017).

Os resultados da análise de regressão linear múltipla mostrou que a única variável que teve influência no desempenho acadêmico foi o domínio "relações sociais". Boa condição no item relações sociais do WHOQOL-bref, pode indicar atitudes positivas entre os colegas da mesma turma, em que um aluno pode dar apoio e suporte ao outro, contribuindo para a melhora do desempenho. Essa característica pode ser um fator importante para futuros profissionais da área da saúde, uma vez que há uma necessidade de maior humanização das relações paciente e profissional (RODRIGUES et al., 2019; SHAREEF et al., 2015). Por outro, lado foi relatado que baixos valores nos domínios "psicológico" e "relações sociais" estavam relacionados com um melhor desempenho acadêmico (MALIBARY et al., 2019). Estudos também mostram que variáveis como condições econômicas, idade, gênero, estado cível do estudante, podem influenciar a QV e no desempenho do aluno (CHAZAN et al., 2015; GAN; YUEN LING, 2019; LANGAME et al., 2016; MOURA et al., 2016; SAUPE et al., 2004; SERINOLLI; NOVARETTI, 2017; SOLIS; LOTUFO-NETO, 2019). No entanto, em nossa pesquisa não foi possível confirmar essa relação. Vários fatores poderiam explicar as diferenças entre os resultados, com por exemplo os diferentes currículos, cursos e condições sócio-político-cultural (CHAZAN et al., 2015; GAN; YUEN LING, 2019; MORITZ et al., 2016; MOURA et al., 2016; RODRIGUES et al., 2019; SHAREEF et al., 2015).

O presente estudo apresenta algumas limitações como por exemplo a amostra pequena e de conveniência, em que utilizamos apenas duas turmas de um mesmo curso de graduação, durante um semestre em uma instituição de ensino pública. Assim, os resultados podem não ser representativos dos cursos de Odontologia que envolvem outras instituições públicas ou privadas, inseridas em regiões com diferentes características sócio-econômicas. O questionário WHOQOL-bref apresenta certa limitação, uma vez que é um instrumento de autorrelato o que pode gerar viés de resposta. Outra limitação do presente estudo, está na complexidade em avaliar o real rendimento acadêmico do universitário, uma vez que, assim como a QV, o desempenho de um estudante é influenciado por distintos fatores. Desta forma, deve-se ter um certo cuidado na extrapolação dos resultados. Estudos utilizando diferentes séries e cursos por períodos mais longos de acompanhamento devem ser considerados.

A partir de resultados da avaliação da QV de estudantes é possível obter informações que podem auxiliar as instituições a promover ações que melhorem o rendimento. Sendo possível a detecção precoce das dificuldades vivenciadas pelos alunos e podem cooperar com o delineamento de estratégias que beneficiem a busca por soluções para os conflitos que incidem na QV, fornecendo suporte físico, emocional e psicológico.

\section{Conclusão}

A partir dos resultados obtidos foi possível concluir que os homens apresentam melhores indicadores de qualidade de vida que as mulheres, principalmente nas facetas relacionadas aos domínios "físico" e "psicológico".

Não foram encontradas diferenças no rendimento acadêmico dos alunos, sendo 
que, o domínio "relações sociais" pode influenciar este parâmetro.

\section{Agradecimentos}

O presente trabalho foi realizado com apoio da Coordenação de Aperfeiçoamento de Pessoal de Nível Superior - Brasil (CAPES) - Código de Financiamento 001.

Ao Conselho Nacional de Desenvolvimento Científico e Tecnológico - CNPq, pela concessão de bolsa através do Programa Institucional de Bolsas de Iniciação Científica $\mathrm{PIBIC/UEPG.}$

\section{Referências}

ABOSHAIQAH, A. E.; CRUZ, J. P. Quality of Life and Its predictors among nursing students in Saudi Arabia. Journal of Holistic Nursing, v. 37, n. 2, p. 200-208, jun. 2019.

ANDRE, A.; PIERRE, G. C.; MCANDREW, M. Quality of life among dental students: a survey study. Journal of Dental Education, v. 81, n. 10, p. 1164-1170, oct. 2017.

CHAZAN, A. C.; CAMPOS, M. R.; PORTUGAL, F. B. Quality of life of medical students at the State University of Rio de Janeiro (UERJ), measured using Whoqol-bref: a multivariate analysis. Ciência e Saúde Coletiva, v. 20, n. 2, p. 547-556, feb. 2015.

COSTA, D. G.; et al. Quality of life and eating attitudes of health care students. Revista Brasileira de Enfermagem, v. 71, n. suppl 4., p. 1642-1649, 2018.

CRUZ, J. P.; et al. Quality of life of nursing students from nine countries: A cross-sectional study. Nurse Education Today, v. 66, p. 135-142, jul. 2018.

FLECK, M. P.; et al. Aplicação da versão em português do instrumento abreviado de avaliação da qualidade de vida "WHOQOL-bref". Revista de Saúde Pública, v. 34, n. 2, p. 178-183, apr. 2000.

GAN, G. G.; YUEN LING, H. Anxiety, depression and quality of life of medical students in Malaysia. The Medical Journal of Malaysia, v. 74, n. 1, p. 57-61, feb. 2019.

LANGAME, A. P.; et al. Qualidade de vida do estudante universitário e o rendimento acadêmico. Revista Brasileira em Promoção da Saude, v. 29, n. 3, p. 313-325, jul./set. 2016.

MALIBARY, $\mathrm{H}$; ; et al. Quality of Life (QoL) among medical students in Saudi Arabia: a study using the WHOQOL-BREF instrument. BMC Medical Education, v. 19, n. 1, p. 344, sep 9. 2019.

MORITZ, A. R.; et al. Quality of life of undergraduate nursing students at a Brazilian public university. Investigación y Educación en Enfermería, v. 34, n. 3, p. 564-572, oct. 2016.

MOURA, I. H.; et al. Quality of life of undergraduate nursing students. Revista Gaúcha de Enfermagem, v. 37, n. 2, p. e55291, jun. 2016. 
PEDROSO, B.; et al. Cálculo dos escores e estatística descritiva do WHOQOL-bref através do Microsoft Excel. Revista Brasileira de Qualidade de Vida, v. 2, n. 1, p. 31-36, jan./jun. 2010.

RODRIGUES, M. I. Q.; et al. Stress factors and quality of life of dental students. Revista da ABENO, v. 19, n. 1, p. 49-57, 2019.

SAUPE, R. S.; et al. Qualidade de vida dos acadêmicos de enfermagem. Revista LatinoAmericana de Enfermagem, v. 12, n. 4, p. 636-642, jul./aug. 2004.

SERINOLLI, M. I.; NOVARETTI, M. C. Z. A cross-sectional study of sociodemographic factors and their influence on quality of life in medical students at Sao Paulo, Brazil. PLoS One, v. 12, n. 7, p. e0180009, jul. 2017.

SHAREEF, M. A.; et al. The interplay between academic performance and quality of life among preclinical students. BMC Medical Education, v. 15, p. 193, oct 31. 2015.

SOLIS, A. C.; LOTUFO-NETO, F. Predictors of quality of life in Brazilian medical students: a systematic review and meta-analysis. Brazilian Journal of Psychiatry, v. pii: S151644462019005007104, apr 15. 2019.

VICTOR, F. F.; et al. Quality of life among university students with premenstrual syndrome. Revista Brasileira de Ginecologia e Obstetrícia, v. 41, n. 5, p. 312-317, may. 2019.

ZHANG, Y.; et al. Quality of life of medical students in China: a study using the WHOQOLBREF. PLoS One, v. 7, n. 11, p. e49714, 2012. 